\title{
On the mean value of the force operator for 1D particles in the step potential
}

\author{
Salvatore De Vincenzo*10 \\ ${ }^{1}$ Universidad Central de Venezuela, Facultad de Ciencias, Escuela de Física, Caracas, Venezuela.
}

Received on October 01, 2020. Revised on January 17, 2021. Accepted on January 18, 2021.

\begin{abstract}
In the one-dimensional Klein-Fock-Gordon theory, the probability density is a discontinuous function at the point where the step potential is discontinuous. Thus, the mean value of the external classical force operator cannot be calculated from the corresponding formula of the mean value. To resolve this issue, we obtain this quantity directly from the Klein-Fock-Gordon equation in Hamiltonian form, or the Feshbach-Villars wave equation. Not without surprise, the result obtained is not proportional to the average of the discontinuity of the probability density but to the size of the discontinuity. In contrast, in the one-dimensional Schrödinger and Dirac theories this quantity is proportional to the value that the respective probability density takes at the point where the step potential is discontinuous. We examine these issues in detail in this paper. The presentation is suitable for the advanced undergraduate level.
\end{abstract}

Keywords: Schrödinger wave equation, Klein-Fock-Gordon wave equation, Dirac wave equation, the external classical force operator, boundary conditions.

\section{Introduction}

Let us consider a one-dimensional quantum particle in the external finite step electric potential, i.e.,

$$
\phi(x)=V_{0} \Theta(x),
$$

where $x \in \mathbb{R}, V_{0}=$ const, and $\Theta(x)$ is the Heaviside step function $(\Theta(x>0)=1$ and $\Theta(x<0)=0)$. Let us suppose that the wave function associated with the particle, $\Psi=\Psi(x, t)$, can be normalizable, that is, $\Psi(x \rightarrow \pm \infty, t)=0$. In fact, we will suppose that the norm of $\Psi$ is equal to one, i.e., $\|\Psi\|^{2} \equiv\langle\Psi, \Psi\rangle=1$, where $\langle$,$\rangle denotes a specific scalar product (as we will see$ below). Note: in the Klein-Fock-Gordon case, this norm could also be equal to -1 (see, for example, Ref. 1]).

Now, let us write the one-dimensional Schrödinger, Klein-Fock-Gordon, and Dirac wave equations, in Hamiltonian form:

$$
\mathrm{i} \hbar \frac{\partial}{\partial t} \Psi=\hat{\mathrm{h}}_{\mathrm{A}} \Psi, \quad \mathrm{A}=\mathrm{S}, \mathrm{KFG}, \mathrm{D},
$$

where

$$
\hat{\mathrm{h}}_{\mathrm{S}}=-\frac{\hbar^{2}}{2 \mathrm{~m}} \frac{\partial^{2}}{\partial x^{2}}+\phi(x)
$$

is the Schrödinger Hamiltonian operator;

$$
\hat{\mathrm{h}}_{\mathrm{KFG}}=-\frac{\hbar^{2}}{2 \mathrm{~m}}\left(\hat{\tau}_{3}+\mathrm{i} \hat{\tau}_{2}\right) \frac{\partial^{2}}{\partial x^{2}}+\mathrm{m} c^{2} \hat{\tau}_{3}+\phi(x),
$$

\footnotetext{
${ }^{*}$ Correspondence email address: salvatore.devincenzo@ucv.ve
}

is -let us say- the Klein-Fock-Gordon Hamiltonian operator (where $\hat{\tau}_{3}=\left[\begin{array}{cc}1 & 0 \\ 0 & -1\end{array}\right]$ and $\hat{\tau}_{2}=\left[\begin{array}{cc}0 & -\mathrm{i} \\ \mathrm{i} & 0\end{array}\right]$ ) and the respective wave equation is called the FeshbachVillars equation [1-4], and

$$
\hat{\mathrm{h}}_{\mathrm{D}}=-\mathrm{i} \hbar c \hat{\alpha} \frac{\partial}{\partial x}+\mathrm{m} c^{2} \hat{\beta}+\phi(x),
$$

is the Dirac Hamiltonian operator (where $\hat{\alpha}$ and $\hat{\beta}$ are the $2 \times 2$ Dirac matrices, which satisfy the relations $\hat{\alpha}^{2}=$ $\hat{\beta}^{2}=\hat{1}$ and $\left.\hat{\alpha} \hat{\beta}+\hat{\beta} \hat{\alpha}=\hat{0}\right)$.

Certainly, the operator $\hat{\mathrm{h}}_{\mathrm{S}}$ acts on one-component wave functions, and the scalar product for these wave functions is the usual one, i.e., $\langle\Psi, \Phi\rangle_{\mathrm{S}}=\int_{\mathbb{R}} \mathrm{d} x \Psi^{*} \Phi$ (the asterisk * denotes the complex conjugate, as usual). Also, $\|\Psi\|_{\mathrm{S}}^{2} \equiv\langle\Psi, \Psi\rangle_{\mathrm{S}}=\int_{\mathbb{R}} \mathrm{d} x \varrho_{\mathrm{S}}$, where $\varrho_{\mathrm{S}}=\varrho_{\mathrm{S}}(x, t)=$ $|\Psi(x, t)|^{2}$ is the Schrödinger probability density. On the other hand, the operator $\hat{\mathrm{h}}_{\mathrm{KFG}}$ acts on two-component

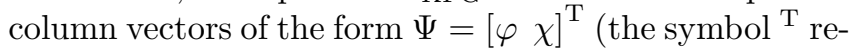
presents the transpose of a matrix). In this case, the scalar product must be defined as $\langle\Psi, \Phi\rangle_{\mathrm{KFG}}=\int_{\mathbb{R}} \mathrm{d} x \Psi^{\dagger} \hat{\tau}_{3} \Phi$ (the symbol $\dagger^{\dagger}$ denotes the Hermitian conjugate, or the adjoint, of a matrix and an operator) [1-4]. Also, $\|\Psi\|_{\mathrm{KFG}}^{2} \equiv\langle\Psi, \Psi\rangle_{\mathrm{KFG}}=\int_{\mathbb{R}} \mathrm{d} x \varrho_{\mathrm{KFG}}$, where $\varrho_{\mathrm{KFG}}=$ $\varrho_{\mathrm{KFG}}(x, t)=\Psi^{\dagger}(x, t) \hat{\tau}_{3} \Psi(x, t)=|\varphi(x, t)|^{2}-|\chi(x, t)|^{2}$ is -let us say- the Klein-Fock-Gordon probability density, although we know that calling this quantity probability density is not absolutely correct (this is because it is not positive definite), see, for example, Refs. [14]. Likewise, the operator $\hat{\mathrm{h}}_{\mathrm{D}}$ acts on two-component wave functions of the form $\Psi=\left[\begin{array}{ll}\psi_{1} & \psi_{2}\end{array}\right]^{\mathrm{T}}$, and the scalar 
product for these wave functions is given by $\langle\Psi, \Phi\rangle_{\mathrm{D}}=$ $\int_{\mathbb{R}} \mathrm{d} x \Psi^{\dagger} \Phi$. Also, $\|\Psi\|_{\mathrm{D}}^{2} \equiv\langle\Psi, \Psi\rangle_{\mathrm{D}}=\int_{\mathbb{R}} \mathrm{d} x \varrho_{\mathrm{D}}$, where $\varrho_{\mathrm{D}}=\varrho_{\mathrm{D}}(x, t)=\Psi^{\dagger}(x, t) \Psi(x, t)=\left|\psi_{1}(x, t)\right|^{2}+\left|\psi_{2}(x, t)\right|^{2}$ is the Dirac probability density. Finally, as is known, Hamiltonians $\hat{\mathrm{h}}_{\mathrm{S}}$ and $\hat{\mathrm{h}}_{\mathrm{D}}$ are Hermitian, or formally selfadjoint, differential operators, i.e., $\hat{\mathrm{h}}_{\mathrm{S}}=\hat{\mathrm{h}}_{\mathrm{S}}^{\dagger}$ and $\hat{\mathrm{h}}_{\mathrm{D}}=$ $\hat{\mathrm{h}}_{\mathrm{D}}^{\dagger}$, thus, $\left\langle\Psi, \hat{\mathrm{h}}_{\mathrm{A}} \Phi\right\rangle_{\mathrm{A}}=\left\langle\hat{\mathrm{h}}_{\mathrm{A}} \Psi, \Phi\right\rangle_{\mathrm{A}}(\mathrm{A}=\mathrm{S}, \mathrm{D})$. Because $\hat{\mathrm{h}}_{\mathrm{KFG}}=\hat{\tau}_{3} \hat{\mathrm{h}}_{\mathrm{KFG}}^{\dagger} \hat{\tau}_{3}$, the Hamiltonian $\hat{\mathrm{h}}_{\mathrm{KFG}}$ is called pseudo-Hermitian, or formally pseudo-self-adjoint, i.e., $\left\langle\Psi, \hat{\mathrm{h}}_{\mathrm{KFG}} \Phi\right\rangle_{\mathrm{KFG}}=\left\langle\hat{\mathrm{h}}_{\mathrm{KFG}} \Psi, \Phi\right\rangle_{\mathrm{KFG}}$.

Let us think about obtaining the average value (or the mean value) of the external classical force operator,

$$
\hat{f}=-\mathrm{d} \phi(x) / \mathrm{d} x=-V_{0} \delta(x),
$$

directly from each wave equation $(\delta(x)=\mathrm{d} \Theta(x) / \mathrm{d} x$ is the Dirac delta function), i.e., let us think about obtaining the following three quantities: $\langle\hat{f}\rangle_{\mathrm{A}}=\int_{\mathbb{R}} \mathrm{d} x \hat{f} \varrho_{\mathrm{A}}$, with $\mathrm{A}=\mathrm{S}, \mathrm{KFG}, \mathrm{D}$, directly from the respective wave equation (even knowing that the calculation of these quantities from the previous formula seems absolutely immediate). Certainly, the procedure to obtain each average value depends on the respective equation, although all three are similar procedures. For example, if the Schrödinger equation is considered, the procedure is as follows: (a) multiply the wave equation for $\Psi$ by $\partial \Psi^{*} / \partial x$ and the equation for $\Psi^{*}$ by $\partial \Psi / \partial x$, and then sum the two resulting equations; (b) integrate each term of the result obtained in (a) around $x=0$. Then, we obtain the following result:

$$
0=-\left.\frac{\hbar^{2}}{2 \mathrm{~m}}\left[\left|\Psi_{x}(x, t)\right|^{2}\right]\right|_{0_{-}} ^{0+}+\left.\left[\phi(x) \varrho_{\mathrm{S}}(x, t)\right]\right|_{0-} ^{0+}+\langle\hat{f}\rangle_{\mathrm{S}},
$$

where we use hereafter the notation $\left.[g]\right|_{0-} ^{0+} \equiv g(0+, t)-$ $g(0-, t)$ with $0 \pm \equiv \lim _{\epsilon \rightarrow 0}(0 \pm \epsilon)$, and also $\Psi_{x} \equiv$ $\partial \Psi / \partial x$. For the step potential given in Eq. (1), we have that $\Psi(0+, t)=\Psi(0-, t) \equiv \Psi(0, t)$ and $\Psi_{x}(0+, t)=$ $\Psi_{x}(0-, t) \equiv \Psi_{x}(0, t)$, i.e., $\Psi(x, t)$ and $\Psi_{x}(x, t)$, and therefore, $\varrho_{\mathrm{S}}(x, t)$, are continuous functions at $x=0$. We finally obtain the result

$$
\langle\hat{f}\rangle_{\mathrm{S}}=-V_{0} \varrho_{\mathrm{S}}(0, t),
$$

which is also obtained immediately from the formula to calculate the average value of the operator $\hat{f}$ in the Schrödinger case, namely, $\langle\hat{f}\rangle_{\mathrm{S}}=$ $-V_{0} \int_{\mathbb{R}} \mathrm{d} x \delta(x) \varrho_{\mathrm{S}}(x, t)=-V_{0} \varrho_{\mathrm{S}}(0, t)$. In the latter result we used Eq. (6) and the defining property of the Dirac delta function, which is valid for continuous functions at the single point where the Dirac delta is infinite. It is worth noting that, the relation we have written in Eq. (7) gives us the average value of the external classical force operator even when $V_{0} \rightarrow \infty$ (see appendix $\mathrm{A}$ ).

Similarly, if the Dirac equation is considered, the procedure is as follows: (a) multiply (properly) the wave equation for $\Psi$ by $\partial \Psi^{\dagger} / \partial x$ and the equation for $\Psi^{\dagger}$ by $\partial \Psi / \partial x$, and then sum the two resulting equations; (b) integrate each term of the resulting expression obtained in (a) over $x=0$. Then, we obtain the following result:

$0=\left.\mathrm{m} c^{2}\left[\Psi^{\dagger}(x, t) \hat{\beta} \Psi(x, t)\right]\right|_{0-} ^{0+}+\left.\left[\phi(x) \varrho_{\mathrm{D}}(x, t)\right]\right|_{0_{-}} ^{0+}+\langle\hat{f}\rangle_{\mathrm{D}}$.

For the potential step in Eq. (1), we have just that $\Psi(0+, t)=\Psi(0-, t) \equiv \Psi(0, t)$, thus, $\Psi^{\dagger}(x, t) \hat{\beta} \Psi(x, t)$ and $\varrho_{\mathrm{D}}(x, t)$ are also continuous functions at $x=0$, and we obtain the result

$$
\langle\hat{f}\rangle_{\mathrm{D}}=-V_{0} \varrho_{\mathrm{D}}(0, t)
$$

Certainly, from the formula to calculate the average value of the operator $\hat{f}$ in the Dirac case, the result in Eq. 10 can also be obtained immediately, namely, $\langle\hat{f}\rangle_{\mathrm{D}}=-V_{0} \int_{\mathbb{R}} \mathrm{d} x \delta(x) \varrho_{\mathrm{D}}(x, t)=-V_{0} \varrho_{\mathrm{D}}(0, t)$. Again, in the latter result we used Eq. (6) and the key property of the Dirac delta function (remember that the latter property only works for functions that are continuous at $x=0$ ). Incidentally, in Ref. [5], the Ehrenfest theorem for the one-dimensional Dirac particle in the finite step potential was studied, and it was demonstrated that the formal time derivative of the mean value of the one-dimensional (Dirac) momentum operator, $\hat{\mathrm{p}}=$ $-\mathrm{i} \hbar \hat{1} \partial / \partial x$, is precisely $\langle\hat{f}\rangle_{\mathrm{D}}$. The result given in Eq. (9) was also obtained in the same reference.

\section{The mean value of $\hat{f}$ in the Klein-Fock-Gordon case}

Now, we consider the Hamiltonian form of the KleinFock-Gordon equation in one spatial dimension, or the one-dimensional first order in time Klein-Fock-Gordon equation, or the one-dimensional Feshbach-Villars wave equation. The procedure to obtain the average value of the operator $\hat{f}$ in Eq. (6) from the latter equation is as follows: (a) multiply (properly) the equation for $\Psi$ by $\left(\partial \Psi^{\dagger} / \partial x\right) \hat{\tau}_{3}$ and the equation for $\Psi^{\dagger}$ by $\hat{\tau}_{3} \partial \Psi / \partial x$, and then sum the two resulting equations; (b) integrate each term of the resulting expression obtained in (a) across $x=0$. Thus, we obtain

$$
\begin{aligned}
0= & -\left.\frac{\hbar^{2}}{2 \mathrm{~m}}\left[\Psi_{x}^{\dagger}(x, t)\left(\hat{1}+\hat{\tau}_{1}\right) \Psi_{x}(x, t)\right]\right|_{0-} ^{0+} \\
& +\left.\mathrm{m} c^{2}\left[\Psi^{\dagger}(x, t) \Psi(x, t)\right]\right|_{0-} ^{0+} \\
& +\left.\left[\phi(x) \varrho_{\mathrm{KFG}}(x, t)\right]\right|_{0-} ^{0+}+\langle\hat{f}\rangle_{\mathrm{KGF}}
\end{aligned}
$$

(where $\hat{\tau}_{1}=\left[\begin{array}{ll}0 & 1 \\ 1 & 0\end{array}\right]$ ). For a discontinuous potential at $x=0$, such as that given in Eq. (1), we have to impose the following boundary conditions: $\psi(0+, t)=$ $\psi(0-, t) \equiv \psi(0, t)$ and $\psi_{x}(0+, t)=\psi_{x}(0-, t) \equiv \psi_{x}(0, t)$, where $\psi=\psi(x, t)$ is the solution of the one-dimensional Klein-Fock-Gordon wave equation in its standard form, or the second order in time Klein-Fock-Gordon equation 
in one spatial dimension [6], namely,

$$
\left[\mathrm{i} \hbar \frac{\partial}{\partial t}-\phi(x)\right]^{2} \psi=\left[-\hbar^{2} c^{2} \frac{\partial^{2}}{\partial x^{2}}+\left(\mathrm{m} c^{2}\right)^{2}\right] \psi
$$

(in fact, the latter equation involves second as well as first time derivatives). The continuity of $\psi$ and $\psi_{x}$ at $x=0$ implies that the Klein-Fock-Gordon probability current density is also continuous there (see, for example, Refs. 3, 4]). In $(3+1)$ dimensions, this result is also true if there is no potential vector, and also if it is different from zero but continuous at $x=0$. Because the current and probability densities satisfy the usual continuity equation, this last result and the continuity of the probability current density at $x=0$ imply that the integral of the probability density over the small interval around the point $x=0$ does not change in time (what is an expected result). Incidentally, in Ref. [10, among other things, it was verified that the stationary solutions of Eq. (12) with the step potential given in Eq. (1), and also its first spatial derivatives, must be continuous at $x=0$. In fact, in that reference, the stationary solutions of the Klein-Fock-Gordon equation with an smooth potential that tends to the step potential after taking a limit were first obtained, then, by taking the same limit on these solutions, the result in question was verified. Also, the relation between $\psi$ (a one-component wave function) and $\Psi$ (a two-component column vector), is given by

$$
\Psi=\left[\begin{array}{l}
\varphi \\
\chi
\end{array}\right]=\frac{1}{2}\left[\begin{array}{l}
\psi+\frac{1}{\mathrm{~m} c^{2}}\left(\mathrm{i} \hbar \frac{\partial}{\partial t}-\phi\right) \psi \\
\psi-\frac{1}{\mathrm{~m} c^{2}}\left(\mathrm{i} \hbar \frac{\partial}{\partial t}-\phi\right) \psi
\end{array}\right]
$$

and from the latter expression we obtain

$$
\psi=\varphi+\chi, \quad \text { and } \quad \frac{1}{\mathrm{~m} c^{2}}\left(\mathrm{i} \hbar \frac{\partial}{\partial t}-\phi\right) \psi=\varphi-\chi .
$$

These relations can be used to write $\varrho_{\mathrm{KFG}}=\Psi^{\dagger} \hat{\tau}_{3} \Psi=$ $|\varphi|^{2}-|\chi|^{2}$ in terms of $\psi$. In effect, the following wellknown result is obtained:

$$
\varrho_{\mathrm{KFG}}=\frac{\mathrm{i} \hbar}{2 \mathrm{~m} c^{2}}\left(\psi^{*} \dot{\psi}-\psi \dot{\psi}^{*}\right)-\frac{\phi}{\mathrm{m} c^{2}} \psi^{*} \psi
$$

where $\dot{\psi} \equiv \partial \psi / \partial t$ (see, for example, Ref. [3]).

Because the potential in Eq. (1) vanishes for $x<0$, and has the value $V_{0}$ for $x>0$, we can write the following relations from Eq. 13):

$$
\Psi=\frac{1}{2}\left[\begin{array}{l}
\psi+\frac{\mathrm{i} \hbar}{\mathrm{m} c^{2}} \dot{\psi} \\
\psi-\frac{\mathrm{i} \hbar}{\mathrm{m} c^{2}} \dot{\psi}
\end{array}\right] \quad\left(\Rightarrow \Psi_{x}=\frac{1}{2}\left[\begin{array}{c}
\psi_{x}+\frac{\mathrm{i} \hbar}{\mathrm{m} c^{2}} \dot{\psi}_{x} \\
\psi_{x}-\frac{\mathrm{i} \hbar}{\mathrm{m} c^{2}} \dot{\psi}_{x}
\end{array}\right]\right)
$$

for $x<0$, and

$$
\begin{aligned}
\Psi=\frac{1}{2}\left[\begin{array}{l}
\psi+\frac{\mathrm{i} \hbar}{\mathrm{m} c^{2}} \dot{\psi}-\frac{V_{0}}{\mathrm{~m} c^{2}} \psi \\
\psi-\frac{\mathrm{i} \hbar}{\mathrm{m} c^{2}} \dot{\psi}+\frac{V_{0}}{\mathrm{~m} c^{2}} \psi
\end{array}\right] \\
\left(\Rightarrow \Psi_{x}=\frac{1}{2}\left[\begin{array}{l}
\psi_{x}+\frac{\mathrm{i} \hbar}{\mathrm{m} c^{2}} \dot{\psi}_{x}-\frac{V_{0}}{\mathrm{~m} c^{2}} \psi_{x} \\
\psi_{x}-\frac{\mathrm{i} \hbar}{\mathrm{m} c^{2}} \dot{\psi}_{x}+\frac{V_{0}}{\mathrm{~m} c^{2}} \psi_{x}
\end{array}\right]\right),
\end{aligned}
$$

for $x>0$ (we also have that $\Psi_{x}=\left[\begin{array}{ll}\varphi_{x} & \chi_{x}\end{array}\right]^{\mathrm{T}}$ ). Thus, from Eqs. (16) and (17), and using the continuity of $\psi$ and $\psi_{x}$ at $x=0$, we obtain the following matricial boundary conditions:

$$
\Psi(0+, t)=\Psi(0-, t)+\frac{1}{2} \frac{V_{0}}{\mathrm{~m} c^{2}}\left[\begin{array}{l}
-1 \\
+1
\end{array}\right] \psi(0, t)
$$

and

$$
\Psi_{x}(0+, t)=\Psi_{x}(0-, t)+\frac{1}{2} \frac{V_{0}}{\mathrm{~m} c^{2}}\left[\begin{array}{l}
-1 \\
+1
\end{array}\right] \psi_{x}(0, t) .
$$

Thus, we have that the column vector $\Psi$ and its first spatial derivative $\Psi_{x}$ are discontinuous functions at $x=0$. Likewise, the Klein-Fock-Gordon probability density is also discontinuous at $x=0$. Precisely, from Eq. 15 we obtain the following result pertaining to this discontinuity:

$$
\varrho_{\mathrm{KFG}}(0+, t)=\varrho_{\mathrm{KFG}}(0-, t)-\frac{V_{0}}{\mathrm{~m} c^{2}} \psi^{*}(0, t) \psi(0, t) .
$$

Incidentally, the boundary conditions in Eqs. (18) and (19) can be written in a way reminiscent of the periodic boundary condition (i.e., thinking in a real line with the origin excluded, or a hole, between $x=0-$ and $x=0+$ ), namely,

$$
\left(\hat{\tau}_{3}+\mathrm{i} \hat{\tau}_{2}\right) \Psi(0+, t)=\left(\hat{\tau}_{3}+\mathrm{i} \hat{\tau}_{2}\right) \Psi(0-, t),
$$

and

$$
\left(\hat{\tau}_{3}+\mathrm{i} \hat{\tau}_{2}\right) \Psi_{x}(0+, t)=\left(\hat{\tau}_{3}+\mathrm{i} \hat{\tau}_{2}\right) \Psi_{x}(0-, t),
$$

respectively; hence, $\left(\hat{\tau}_{3}+\mathrm{i} \hat{\tau}_{2}\right) \Psi$ and $\left(\hat{\tau}_{3}+\mathrm{i} \hat{\tau}_{2}\right) \Psi_{x}$ are continuous functions at $x=0$. Clearly, this result is obtained by multiplying Eqs. (18) and 19 from the left by the matrix $\hat{\tau}_{3}+\mathrm{i} \hat{\tau}_{2}$. The latter two boundary conditions can also be obtained directly from the one-dimensional Feshbach-Villars wave equation (see appendix B).

Finally, to obtain $\langle\hat{f}\rangle_{\mathrm{KFG}}$ from the formula (11), we must compute the three boundary terms that are present there. Thus, we can report the following results:

$$
\left.\left[\phi(x) \varrho_{\mathrm{KFG}}(x, t)\right]\right|_{0-} ^{0+}=V_{0} \varrho_{\mathrm{KFG}}(0+, t),
$$

which is obtained immediately. Additionally,

$$
\begin{aligned}
\mathrm{m} c^{2} & {\left.\left[\Psi^{\dagger}(x, t) \Psi(x, t)\right]\right|_{0-} ^{0+} } \\
& =-\frac{V_{0}}{2}\left[\varrho_{\mathrm{KFG}}(0+, t)+\varrho_{\mathrm{KFG}}(0-, t)\right],
\end{aligned}
$$

where we have used the result given in Eq. (18), the relation on the right side of Eq. (14), and Eq. (15), both evaluated at $x=0-$, and Eq. 20). Likewise,

$$
-\left.\frac{\hbar^{2}}{2 \mathrm{~m}}\left[\Psi_{x}^{\dagger}(x, t)\left(\hat{1}+\hat{\tau}_{1}\right) \Psi_{x}(x, t)\right]\right|_{0-} ^{0+}=0,
$$

where we have used the result given in Eq. (19). Then, substituting the three latter results into Eq. (11), we obtain the result

$$
\langle\hat{f}\rangle_{\mathrm{KFG}}=-\frac{V_{0}}{2}\left[\varrho_{\mathrm{KFG}}(0+, t)-\varrho_{\mathrm{KFG}}(0-, t)\right] .
$$


From the formula to calculate the average value of the operator $\hat{f}$ in the Klein-Fock-Gordon case, the result in Eq. 26) cannot be derived. In effect, $\langle\hat{f}\rangle_{\mathrm{KFG}}=$ $\int_{\mathbb{R}} \mathrm{d} x \hat{f} \varrho_{\mathrm{KFG}}(x, t)=-V_{0} \int_{\mathbb{R}} \mathrm{d} x \delta(x) \varrho_{\mathrm{KFG}}(x, t)$, but $\varrho_{\mathrm{KFG}}$ $(x, t)$ is not continuous at $x=0$ (see Eq. (20)). Thus, following this last procedure we could not say anything else, but, as we have seen, the first order in time KleinFock-Gordon equation can determine precisely what is the value of the integral $\int_{\mathbb{R}} \mathrm{d} x \delta \varrho_{\mathrm{KFG}}$. In effect, we can write

$$
\int_{\mathbb{R}} \mathrm{d} x \delta(x) \varrho_{\mathrm{KFG}}(x, t)=\frac{1}{2}\left[\varrho_{\mathrm{KFG}}(0+, t)-\varrho_{\mathrm{KFG}}(0-, t)\right] .
$$

From Eq. 20 we can also write $\langle\hat{f}\rangle_{\mathrm{KFG}}$ in terms of the one-component wave function $\psi$, namely,

$$
\langle\hat{f}\rangle_{\mathrm{KFG}}=+\frac{V_{0}}{2} \frac{V_{0}}{\mathrm{~m} c^{2}} \psi^{*}(0, t) \psi(0, t) .
$$

It is worth mentioning that, the result in Eq. 26 confirms that an integral like the one given in the left side of Eq. 27) is not always equal to the average of the discontinuity of the function that accompanies the Dirac delta in the integral (in this case, the function $\left.\varrho_{\mathrm{KFG}}\right)$, i.e., the right side of Eq. (27) is not necessarily equal to $\frac{1}{2}\left[\varrho_{\mathrm{KFG}}(0+, t)+\varrho_{\mathrm{KFG}}(0-, t)\right]$. For other interesting examples in which similar situations arise, see Refs. [11-13.

Let us finally develop the nonrelativistic approximation of the result in Eq. 26) (or the result in Eq. (28)). In order to do this, we first write the KleinFock-Gordon wave function in the form $\psi(x, t)=$ $\Psi_{\mathrm{S}}(x, t) \exp \left(-\mathrm{im} c^{2} t / \hbar\right)$ (here, $\Psi_{\mathrm{S}}$ is the Schrödinger wave function), and therefore, $\dot{\psi}(x, t)=\left(-\mathrm{im} c^{2} / \hbar\right)$ $\Psi_{\mathrm{S}}(x, t) \exp \left(-\mathrm{im} c^{2} t / \hbar\right)$ (in this limit, we have that $\left.\left|\mathrm{i} \hbar \dot{\Psi}_{\mathrm{S}}\right| \ll \mathrm{m} c^{2}\left|\Psi_{\mathrm{S}}\right|\right)$ [4]. Then, we note that, in the nonrelativistic limit, the Klein-Fock-Gordon probability density given in Eq. 15 reduces to

$$
\varrho_{\mathrm{KFG}}=\varrho_{\mathrm{S}}-\frac{\phi}{\mathrm{m} c^{2}} \varrho_{\mathrm{S}}
$$

(remember that $\varrho_{\mathrm{S}}=\Psi_{\mathrm{S}}^{*} \Psi_{\mathrm{S}}$ ). Using this result, we can also write $\langle\hat{f}\rangle_{\mathrm{KFG}}$ in this limit (see Eq. 26), namely,

$$
\begin{aligned}
\langle\hat{f}\rangle_{\mathrm{KFG}}= & -\frac{V_{0}}{2}\left[\varrho_{\mathrm{S}}(0, t)-\frac{\phi(0+)}{\mathrm{m} c^{2}} \varrho_{\mathrm{S}}(0, t)\right] \\
& +\frac{V_{0}}{2}\left[\varrho_{\mathrm{S}}(0, t)-\frac{\phi(0-)}{\mathrm{m} c^{2}} \varrho_{\mathrm{S}}(0, t)\right] \\
= & +\frac{V_{0}}{2} \frac{V_{0}}{\mathrm{~m} c^{2}} \varrho_{\mathrm{S}}(0, t)
\end{aligned}
$$

(which can also be obtained directly from Eq. (28)). Likewise, using the result in Eq. 29), we can write

$$
\begin{aligned}
\langle\hat{f}\rangle_{\mathrm{KFG}} & =\int_{\mathbb{R}} \mathrm{d} x \hat{f} \varrho_{\mathrm{KFG}} \\
& =\langle\hat{f}\rangle_{\mathrm{S}}+\frac{V_{0}}{\mathrm{~m} c^{2}} \int_{\mathbb{R}} \mathrm{d} x \delta(x) \phi(x) \varrho_{\mathrm{S}}(x, t),
\end{aligned}
$$

but the latter integral cannot be precisely evaluated because $\phi(x)$ is not continuous at $x=0$. Despite that, we decide to define the value of that integral as $\phi(0) \varrho_{\mathrm{S}}(0, t)$, and therefore, the result in Eq. (31) is as follows:

$$
\langle\hat{f}\rangle_{\mathrm{KFG}}=\int_{\mathbb{R}} \mathrm{d} x \hat{f} \varrho_{\mathrm{KFG}}=\langle\hat{f}\rangle_{\mathrm{S}}+\frac{V_{0}}{\mathrm{~m} c^{2}} \phi(0) \varrho_{\mathrm{S}}(0, t) .
$$

Finally, by equating the results given in Eqs. (30) and (32), we obtain

$$
\langle\hat{f}\rangle_{\mathrm{S}}=-\left[\phi(0)-\frac{V_{0}}{2}\right] \frac{V_{0}}{\mathrm{~m} c^{2}} \varrho_{\mathrm{S}}(0, t) .
$$

Certainly, we already know what this last result should be, namely, $\langle\hat{f}\rangle_{\mathrm{S}}=-V_{0} \varrho_{\mathrm{S}}(0, t)$ (Eq. (8)). Thus, in the nonrelativistic limit, $\frac{V_{0}}{2}+\mathrm{m} c^{2}$ should tend to $\phi(0)$. In any case, the latter result can be obtained as follows. First, the one-dimensional Klein-Fock-Gordon wave equation in Hamiltonian form is fully equivalent to the following system of coupled differential equations:

$$
\begin{aligned}
& \mathrm{i} \hbar \frac{\partial}{\partial t} \varphi=-\frac{\hbar^{2}}{2 \mathrm{~m}} \frac{\partial^{2}}{\partial x^{2}}(\varphi+\chi)+\phi \varphi+\mathrm{m} c^{2} \varphi \\
& \mathrm{i} \hbar \frac{\partial}{\partial t} \chi=+\frac{\hbar^{2}}{2 \mathrm{~m}} \frac{\partial^{2}}{\partial x^{2}}(\varphi+\chi)+\phi \chi-\mathrm{m} c^{2} \chi
\end{aligned}
$$

where the top and bottom components of the column vector $\Psi, \varphi$ and $\chi$, are given in terms of $\psi$ in Eq. (13). Then, if we consider the ansatz $\psi=\Psi_{\mathrm{S}} \exp \left(-\mathrm{im} c^{2} t / \hbar\right)$, and therefore, $\dot{\psi}=\left(-\mathrm{im} c^{2} / \hbar\right) \psi$ (the latter in the nonrelativistic limit), we obtain, in this approximation, $\varphi=\left(1-\frac{\phi}{2 m c^{2}}\right) \psi$ (see Eq. (13)). We also have that $\varphi+\chi=\psi$ (see Eq. (14)). Thus, for weak external fields (to lowest order), $\varphi \approx \psi$ satisfies the nonrelativistic Schrödinger equation but with the replacement $\phi+$ $\mathrm{m} c^{2} \rightarrow \phi$ (see the first equation in Eq. (34)), and therefore, $\phi(0)+\mathrm{m} c^{2} \rightarrow \phi(0)$, and also $\frac{V_{0}}{2}+\mathrm{m} c^{2} \rightarrow$ $\phi(0)\left(\equiv \frac{V_{0}}{2}\right)$ (in the last relation, we set the value $\phi \equiv \frac{V_{0}}{2}$ at $x=0)$.

\section{Conclusion}

We have obtained expressions for the mean value of the external classical force operator for a 1D Schrödinger, Klein-Fock-Gordon, and Dirac particle, in the finite step potential. As we have seen, only in the Klein-FockGordon theory this quantity cannot be calculated from the corresponding formula of the mean value. This is because the corresponding probability density is a discontinuous function at the point where the step potential is discontinuous $(x=0)$. However, this quantity can always be calculated from the respective wave equation, which in the latter case is the Feshbach-Villars equation. As we have seen, the result obtained is proportional to the size of the discontinuity of the probability density [the difference $\left.\varrho_{\mathrm{KFG}}(0+, t)-\varrho_{\mathrm{KFG}}(0-, t)\right]$, something that is not expected. In contrast, in the Schrödinger and 
Dirac theories, the result obtained is simply proportional to the value that the respective probability density takes at $x=0$, i.e., this result is proportional to the average of the (non-existent) discontinuity of the probability density [the sum $\varrho_{\mathrm{A}}(0+, t)+\varrho_{\mathrm{A}}(0-, t)=2 \varrho_{\mathrm{A}}(0, t)$, with $\mathrm{A}=\mathrm{S}, \mathrm{D}]$.

As mentioned throughout the paper, the external classical force operator usually appears when studying Ehrenfest's theorem. Here, the most interesting case is when the potential has finite and/or infinite discontinuities. We have seen various studies on this subject in Schrödinger's theory (see, for example, Refs. [14 17] and references therein), and in Dirac's theory [5]. However, in the Klein-Fock-Gordon theory, as far as we know, there are not many studies that involve the mean value of this operator. In fact, the main result of the present article, that is, the result given in Eq. (26), does not seem to have been published before. We believe that our study may be attractive to all those interested in fundamental mathematical aspects of quantum mechanics.

\section{Acknowledgments}

The author would like to thank the anonymous referee for carefully reading the manuscript and for giving such constructive comments an suggestions.

\section{Appendix A}

We want to prove that Eq. (7) can give the average value of the external classical force operator even when $V_{0} \rightarrow \infty$, i.e., for a Schrödinger particle in an infinite step potential $(\phi(0-)=0$ and $\phi(0+)=\infty)$. In effect, in the latter case we have that $\Psi(0+, t)=\Psi_{x}(0+, t)=0$ (this is the Dirichlet boundary condition imposed on $\Psi$ and $\Psi_{x}$ at $\left.x=0+\right)$, and therefore, $\varrho_{\mathrm{S}}(0+, t)=0$, and although we have that $\phi(0+)=\infty$, we also have that $\phi(0+) \Psi(0+, t)=\infty \times 0=0$ (below, we formally prove this last result, but it must be true because the Schrödinger equation must also be verified at $x=0+$ ); thus, from Eq. (7), we obtain

$0=+\frac{\hbar^{2}}{2 \mathrm{~m}}\left|\Psi_{x}(0-, t)\right|^{2}+\langle\hat{f}\rangle_{\mathrm{S}} \Rightarrow\langle\hat{f}\rangle_{\mathrm{S}}=-\frac{\hbar^{2}}{2 \mathrm{~m}}\left|\Psi_{x}(0-, t)\right|^{2}$

Incidentally, in Ref. [18, the latter result was obtained directly from the same procedure presented when deriving Eq. (7), assuming from the beginning that $V_{0} \rightarrow \infty$. On the other hand, in Ref. [14, the result in Eq. A1 was obtained directly from the Ehrenfest theorem for a (free) Schrödinger particle on a half line (in our case, the region $x \in(-\infty, 0])$. Finally, let us now prove that the product $\phi \Psi$ to the right of the point $x=0$ where the potential makes an infinite jump (i.e., at the extensive impenetrable barrier located in the region $x>0)$, vanishes, i.e., $\phi(0+) \Psi(0+, t)=0(=\infty \times 0)$. In effect, integrating the Schrödinger equation (Eq. (2) with $\mathrm{A}=\mathrm{S}$ ) from $x=0-$ to $x=0+$ yields

$$
0=\frac{\hbar^{2}}{2 \mathrm{~m}} \Psi_{x}(0-, t)+\int_{0-}^{0+} \mathrm{d} x \phi(x) \Psi(x, t),
$$

where we have made use of the condition $\Psi_{x}(0+, t)=0$. Clearly, the relation in Eq. A2 is satisfied by writing the following relation:

$$
\phi(x) \Psi(x, t)=-\frac{\hbar^{2}}{2 \mathrm{~m}} \Psi_{x}(0-, t) \delta(x)
$$

(this is because the Dirac delta function satisfies $\left.\int_{0-}^{0+} \mathrm{d} x \delta(x)=1\right)$. Thus, from Eq. (A3), we obtain the desired result,

$$
\phi(0+) \Psi(0+, t)=0,
$$

and also the (expected) result $\phi(0-) \Psi(0-, t)=0$ (remember that $\delta(0+)=\delta(0-)=0$ ), however, $\phi(0) \Psi(0, t)=\infty$ (this is because $\delta(0)=\infty)$. Incidentally, the result given by Eq. A3 was also obtained and discussed in Ref. [19].

\section{Appendix B}

We want to prove that the boundary conditions for $\Psi$ and $\Psi_{x}$ given in Eqs. 21) and 22 arise directly from the Feshbach-Villars equation. In effect, integrating the latter equation (Eq. (2) with $\mathrm{A}=\mathrm{KFG}$ ) from $x=0$ - to $x=0+$ gives

$$
0=-\left.\frac{\hbar^{2}}{2 \mathrm{~m}}\left[\left(\hat{\tau}_{3}+\mathrm{i} \hat{\tau}_{2}\right) \Psi_{x}(x, t)\right]\right|_{0-} ^{0+}+0+0,
$$

from which the boundary condition given in Eq. 22 is obtained. Likewise, integrating the Feshbach-Villars equation first from $x=-\ell$ (with $\ell>0$ ) to $x$ and then once more from $x=0-$ to $x=0+$ gives

$$
\begin{aligned}
\mathrm{i} \hbar \int_{0-}^{0+} & \mathrm{d} x \int_{-\ell}^{x} \mathrm{~d} y \dot{\Psi}(y, t) \\
= & -\left.\frac{\hbar^{2}}{2 \mathrm{~m}}\left[\left(\hat{\tau}_{3}+\mathrm{i} \hat{\tau}_{2}\right) \Psi(x, t)\right]\right|_{0-} ^{0+} \\
& +\frac{\hbar^{2}}{2 \mathrm{~m}}\left(\hat{\tau}_{3}+\mathrm{i} \hat{\tau}_{2}\right) \Psi_{x}(-\ell, t) \int_{0-}^{0+} \mathrm{d} x \\
& +\mathrm{m} c^{2} \int_{0-}^{0+} \mathrm{d} x \int_{-\ell}^{x} \mathrm{~d} y \hat{\tau}_{3} \Psi(y, t) \\
& +\int_{0-}^{0+} \mathrm{d} x \int_{-\ell}^{x} \mathrm{~d} y \phi(y) \Psi(y, t),
\end{aligned}
$$

that is,

$$
0=-\left.\frac{\hbar^{2}}{2 \mathrm{~m}}\left[\left(\hat{\tau}_{3}+\mathrm{i} \hat{\tau}_{2}\right) \Psi(x, t)\right]\right|_{0-} ^{0+}+0+0+0,
$$

from which the boundary condition given in Eq. (21) is obtained. Note that for any discontinuous potential at $x=0$, and not only for the jump potential, we obtain the continuity of $\left(\hat{\tau}_{3}+\mathrm{i} \hat{\tau}_{2}\right) \Psi$ and $\left(\hat{\tau}_{3}+\mathrm{i} \hat{\tau}_{2}\right) \Psi_{x}$ at that point. 


\section{References}

[1] A. Wachter, Relativistic Quantum Mechanics (Springer, Berlin, 2011).

[2] H. Feshbach and F. Villars, Rev. Mod. Phys. 30, 24 (1958).

[3] G. Baym, Lectures on Quantum Mechanics (Westview Press, New York, 1990).

[4] W. Greiner, Relativistic Quantum Mechanics (Springer, Berlin, 2000), 3rd ed.

[5] S. De Vincenzo, J. Phys. Stud. 19, 1003 (2015).

[6] O. Klein, Zeitschrift für Physik 37, 895 (1926).

[7] V. Fock, Zeitschrift für Physik 38, 242 (1926).

[8] W. Gordon, Zeitschrift für Physik 40, 117 (1926).

[9] H. Kragh, Am. J. Phys. 52, 1024 (1984).

[10] M. Merad, L. Chetouani and A. Bounames, Phys. Lett. A 267, 225 (2000).

[11] M.G. Calkin, D. Kiang and Y. Nogami, Am. J. Phys. 55, 737 (1987).

[12] D. Griffiths and S. Walborn, Am. J. Phys. 67, 446 (1999).

[13] F.A.B. Coutinho, Y. Nogami and F.M. Toyama, Revista Brasileira de Ensino de Física 31, 4302 (2009).

[14] S. De Vincenzo, Rev. Mex. Fís. E 59, 84 (2013).

[15] D.S. Rokhsar, Am. J. Phys. 64, 1416 (1996).

[16] S. De Vincenzo, Revista Brasileira de Ensino de Física 35, 2308 (2013)

[17] S. De Vincenzo, Pramana - J. Phys. 80, 797 (2013).

[18] M. Andrews, Am. J. Phys. 76, 236 (2008).

[19] S. De Vincenzo, Rev. Mex. Fís. E 54, 1 (2008). 